\title{
Traumatic rupture of an intracranial dermoid cyst: A case report
}

\section{Uddalok Das ${ }^{1}$, Narayan Pandit ${ }^{2}$}

From ${ }^{1}$ Post Graduate Trainee, ' 2 Professor and Head, Department of Radiodiagnosis, North Bengal Medical College and Hospital, Siliguri, West Bengal, India

Correspondence to: Uddalok Das, Department of Radiodiagnosis, North Bengal Medical College and Hospital, Siliguri, West Bengal, India. E-mail: rikdas05@gmail.com

Received - 27 October $2021 \quad$ Initial Review - 18 November 2021

Accepted - 27 November 2021

\section{ABSTRACT}

Intracranial dermoid cyst is a very rare primary intracranial tumor accounting for $<0.5 \%$ of intracranial masses. They are usually congenital and benign. Rupture of intracranial dermoid may occur spontaneously or with trivial trauma. Most cases of rupture are spontaneous. There are very few reported cases of traumatic rupture of intracranial dermoid cysts in world medical literature. The presentation can range from headache to signs of meningeal irritation and focal neurological deficit. Computed tomography (CT) and magnetic resonance imaging (MRI) of the brain is of paramount importance for early diagnosis and prompt treatment planning which can reduce both morbidity and mortality. The following case report gives an account of a 49-year-old female who presented with complaints of headache after an episode of fall. She was examined and no neurological deficit was found. Examination of the neurological system was within normal limits. She underwent a CT and MRI of the brain and was subsequently diagnosed as traumatic rupture of dermoid cyst based on the findings. She was treated with high-dose steroids until resolution of her symptoms and then discharged with the advice of regular follow-up. This case report highlights the role of CT and MRI in early diagnosis and treatment planning of patients with ruptured dermoid cysts which leads to a decrease in mortality and morbidity and also the ability of the same to rule out other more sinister causes of headache in patients.

Key words: Dermoid, Intracranial, Rupture, Traumatic

$\mathrm{D}$ ermoid cysts are rare intracranial tumors of congenital origin. They are often detected incidentally during computed tomography (CT) or magnetic resonance imaging (MRI) scans of the brain done for other indications [1,2]. They are most commonly seen in the midline. Mostly rupture of a dermoid occurs spontaneously. Rarely however traumatic ruptures have been reported in world medical literature [3-5]. CT and MRI are the best radiological tools to make the diagnosis. CT and MRI both reveal a fatty lesion in the brain. Fat droplets disseminated in the brain are considered to be the most important findings indicating rupture. The presentation can be variable from focal neurological deficits to seizures, visual disturbances, hemiplegia, and chemical meningitis. Treatment is principally neurosurgical however it has to be decided on a case-to-case basis weighing the risks and benefits and may include medical, neurosurgical, or both approaches.

\section{CASE REPORT}

A 49-year-old female presented to the emergency of North Bengal Medical College and Hospital with a headache, after an episode of fall in her bathroom $4 \mathrm{~h}$ ago. She was immediately admitted for further evaluation. There was no history of loss of consciousness or vomiting since the fall. She had no other significant past medical or surgical history. There was no history of such events in the family in past. There on examination, she was alert conscious, and cooperative oriented to time place, and person. Glasgow Coma Scale score was 15/15. All higher functions were normal. Sensory and motor system examinations were within normal limits. No signs of meningeal irritation, neurological deficit, cranial nerve palsy, or increased intracranial pressure were noted. A bedside cerebrospinal fluid (CSF) tap was done which revealed clear fluid which was subsequently sent for analysis. Blood sugar, electrolytes, and a complete hemogram were sent as a part of the routine investigation policy of the institution. All the reports were within normal limits and noncontributory. A non-contrast CT (NCCT) scan of the brain was carried out to rule out any cerebrovascular event.

NCCT of the brain revealed a $36 \mathrm{~mm} \times 5 \mathrm{~mm}$ mixed density area in the bifrontal lobe. A fat density component corresponding to 97 Hounsfield Unit was seen in the lesion. Multiple foci of calcification were noted in the lesion (Figs. 1 and 2). Multiple small fat density foci were seen within the anterior interhemispheric fissure. A tiny fat density focus was seen in the left frontal convexity. Tiny calcifications were scattered along the anterior interhemispheric fissure (Fig. 3). There was no evidence of hemorrhage or stroke in the CT scan. Subsequently, MRI of the brain was done.

MRI of the brain plain and contrast study after administration of intravenous (IV) Gadolinium revealed mildly enhancing 
fatty and calcific lobulated mass, measuring $40 \mathrm{~mm} \times 5 \mathrm{~mm}$ in right frontal lobe extending into left frontal lobe and along the interhemispheric fissure of the frontal lobe with mild perilesional edema. Inferiorly the mass abuts the cribriform plate without extension into the nasal cavity. On T1 weighted image (T1WI) the mass was heterogeneously hyperintense. On T2WI the mass was again hyperintense with a hypointense rim surrounding it (Fig. 4). Few tiny fat intensity areas were seen predominantly along the anterior interhemispheric fissure. The mass was hyperintense on the fluid-attenuated inversion recovery (FLAIR) sequence (Fig. 5) and gradient recalled echo (GRE) sequence showed blooming in the mass and its vicinity (Fig. 6). There was no obvious contrast enhancement after IV gadolinium administration (Fig. 7). No other abnormal signal intensity or structural abnormality was noticed in the brain.

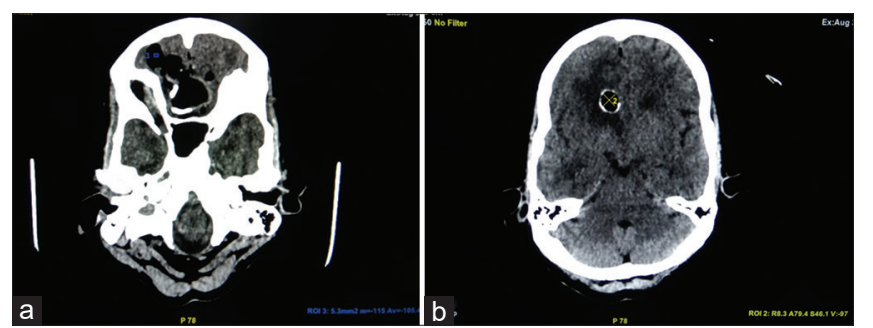

Figure 1: (a) Non-contrast computed tomography (NCCT) brain axial section showing a mixed density lesion in bifrontal lobe with central fat attenuaton (Hounsfield unit [HU]: -105) and peripheral calcification (HU: +322 ), (b) NCCT brain axial section at a higher level showing central fat attenuation (HU: -97) and peripheral calcification (HU: +334)
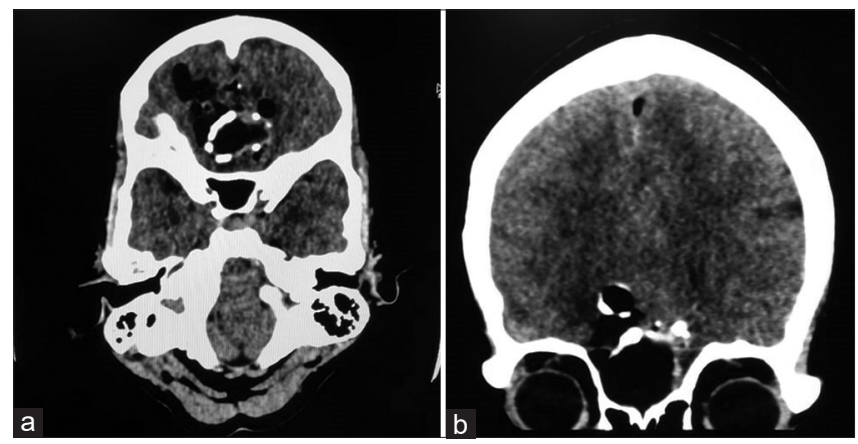

Figure 2: (a) Non-contrast computed tomography (NCCT) brain axial section (b) NCCT brain coronal section both showing a mixed density lesion in bifrontal lobe with scattered fat attenuation and calcific foci
Based on the clinical and radiological findings a diagnosis of intracranial dermoid cyst in right basifrontal lobe with rupture was established. The patient was started on IV steroids and was changed to oral steroids after the $10^{\text {th }}$ day. By 15 days, there was a complete resolution of her symptom and no other new symptom or signs were observed. There was no neurological deficit. The dissemination of fat droplets following the rupture was too extensive to allow for complete removal as judged by the attending neurosurgeon. Furthermore, it is known that many times the dermoid capsules have a dense adherence to the brain parenchyma and vasculature and so complete removal are very difficult. Considering the above points, it was decided by the neurosurgeons to manage the patient conservatively with an option of emergency surgery if new signs or symptoms develop. The patient was discharged with an oral steroid regimen with slow tapering over 3 months. The course of her hospital stay was uneventful. There were no adverse or unexpected events during the hospital stay. She was also asked to attend the Neurosurgery outpatient department for regular follow-up or the emergency reception in case any emergency arises. The plan was to evaluate the patient clinically and with serial MRI brain on follow-up visits as would be deemed fit by the treating neurosurgeon. The patient was explained in detail about the treatment being offered to her, including the rationale for choosing it, its side effects, and all possible outcomes. She was also told about the remote possibility of the development of a squamous cell carcinoma in a dermoid. She was satisfied with the treatment and the regimen was well tolerated by the patient. She agreed to turn up for regular follow-up visits at the hospital.

\section{DISCUSSION}

Dermoid cysts comprise $0.04-0.6 \%$ of all intracranial primary tumors $[1,2]$. They develop during fetal development. Dermal components become trapped intracranially along the lines of embryonic closure and give rise to dermoid cysts. They are ectodermal inclusion cysts of congenital origin. Intracranial dermoid cysts are extra-axial lesions and are most commonly found in the midline. They occur in decreasing frequency in the suprasellar cistern, posterior fossa, and frontonasal region.

Pathologically the wall of the cyst is lined by a thick layer of stratified squamous epithelium. Internal contents vary including sebaceous material, keratin, hair, lipid, and cholesterol material.
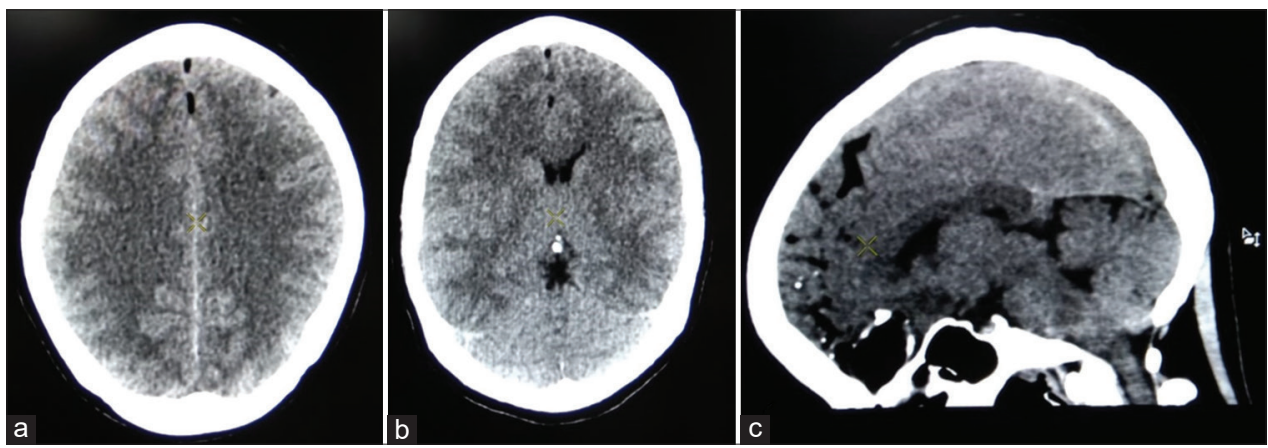

Figure 3: (a) Non-contrast computed tomography (NCCT) brain axial section (b) NCCT brain axial section at a lower level (c) NCCT brain sagittal section showing fat density foci in the anterior interhemispheric fissure 


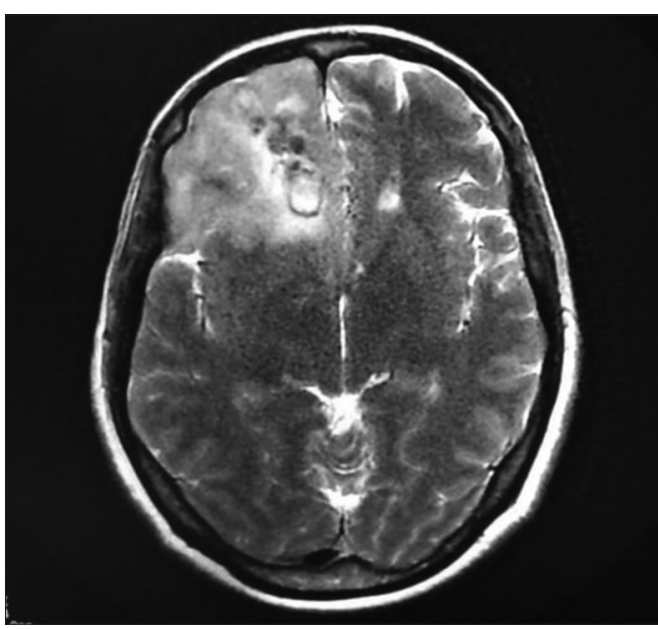

Figure 4: Axial T2 weighted image shows a hyperintense lesion surrounded by a hypo intense rim in right frontal lobe

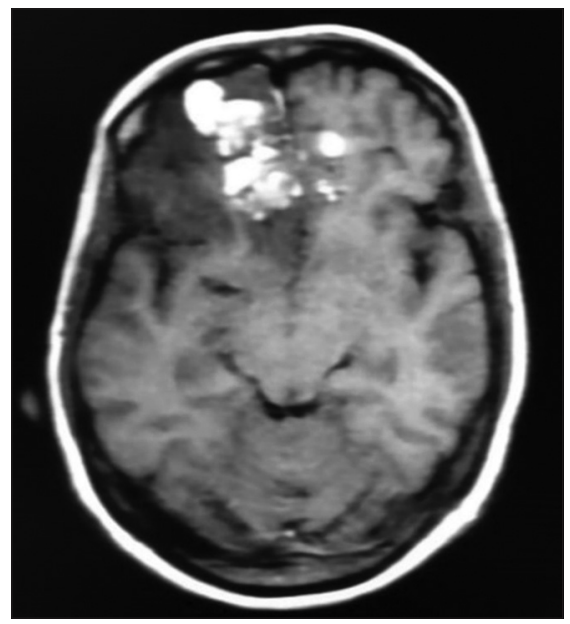

Figure 5: Axial fluid-attenuated inversion recovery sequence showing the lesion is hyperintense

Dermoid cysts grow at a very slow rate and rupture occurs in very few cases. Among the cases of rupture spontaneous rupture is most common. There is a limited number of cases in world medical literature, where rupture is traumatic [3-5].

Patients with a ruptured dermoid cyst may have a varied clinical presentation from a simple headache onto hemiplegia, seizures loss of consciousness, visual disturbance, facial numbness, neck pain, and meningitis. The symptoms depend on the location of the cyst, the mass effect produced due to them, and due to dissemination of contents on rupture. Obstruction of the CSF pathway or occlusion of ventricular outflow path may cause hydrocephalus. Compression over optic chiasma may lead to visual loss. Upon rupture, symptoms may occur shortly or may be delayed for a variable duration. The lipid droplets disseminated into the brain and subarachnoid space due to rupture of the cyst cause irritation of the brain and neurovascular bundles leading to aseptic chemical meningitis.

The radiological investigations include a CT scan and MRI brain. NCCT brain shows a hypodense lesion with fat attenuation. There is peripheral calcification representing calcification of the capsule in $20 \%$ of cases. On rupture, there is dissemination of fat droplets into CSF cisterns and adjoining areas that are visualized

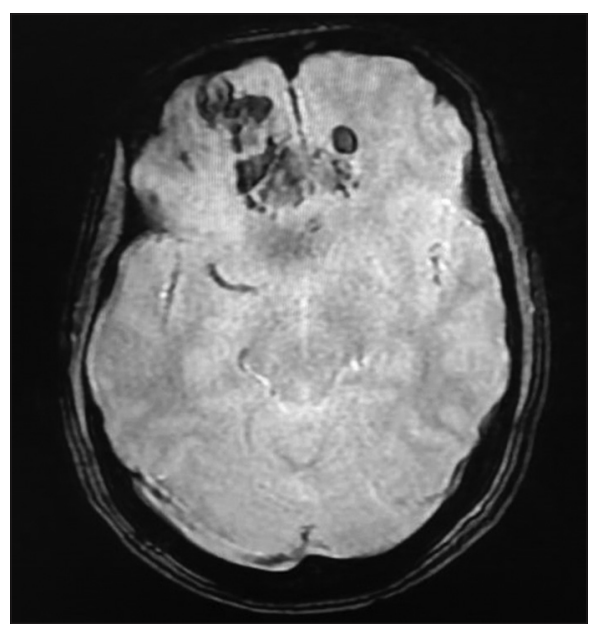

Figure 6: Axial gradient recalled echo sequence showing areas of blooming

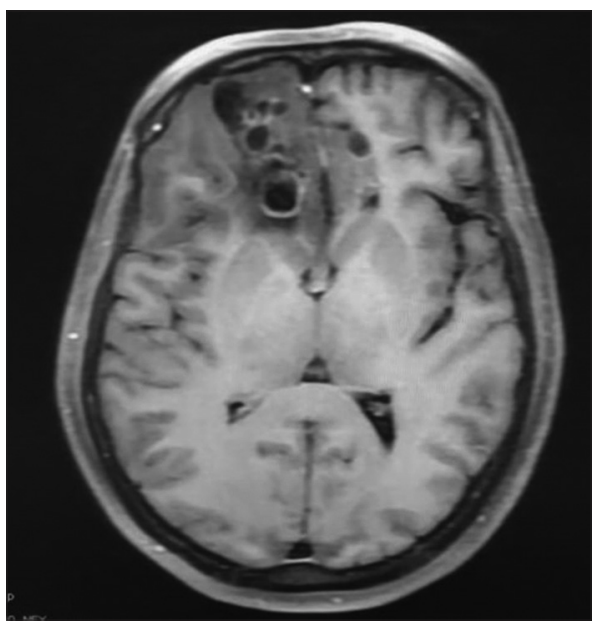

Figure 7: Axial T1 weighted image with fat suppression and contrast shows the lesion is hypointense with no obvious contrast enhancement

as hypodensities. A fat fluid level is sometimes appreciated in the ventricles [6].

MRI is the best investigative modality for making the diagnosis [7]. On MRI the appearance depends on the fat content. The lesions are heterogeneously hyperintense on T1WI. It is the best sequence to detect disseminated fat droplets in the subarachnoid space indicating rupture. Fat suppression confirms the same. On T2WI the lesions are again hyperintense. If hair is present in the cyst there may be a striated laminated appearance on T2WI. Ruptured dermoid cysts are hyperintense on the FLAIR sequence and bloom on the GRE sequence [1]. The typical imaging features matched with the findings in our case. In case the rupture causes meningitis there is a meningeal enhancement on contrast administration which was not seen in our case. Spectroscopy reveals an elevated lipid peak due to the nature of the contents of the dermoid cyst. Fat in the CSF spaces may persist for a long-time rupture [8]. The differential diagnosis of the condition includes epidermoid cysts, lipoma, craniopharyngioma, and teratoma [1].

Treatment includes high doses of IV steroids and surgical removal of the cyst. The risk-benefit ratio of complete surgical removal of the cyst versus an injury to neurovascular structures has 
to be carefully evaluated before deciding on treatment $[4,9,10]$. Medical management is only symptomatic. The definitive management is total excision of the cyst.

Surgery is indicated only in cases where dermoid cysts causing serious neurological deficits [11]. In a retrospective review of 33 patients, Lynch et al. referred that total tumor removal was achieved in 24 cases $(72.7 \%)$. The most common complications occurred due to the dissection of the adherent capsule from the third, sixth, seventh, and eighth cranial nerves, which led to their palsy. However, most of them were transient [12]. Kosuge et al. considered that conservative treatment as an approach should be examined as a therapeutic alternative in cases with a headache or controllable seizure without any visual disturbance [13]. In cases where the rupture has occurred, there is extensive dissemination of fat droplets which are very difficult to remove completely like in our case. Long-term monitoring with serial MRI scans and clinical examinations of patients with extensive disseminated fat particles following rupture of dermoid has not demonstrated progression or movement of the fat or new-onset neurological deterioration [14]. In previously documented cases of traumatic rupture of intracranial dermoid cysts, most have opted for surgical intervention and one case was managed conservatively with observation and regular follow-ups $[2,4,9,15,16]$.

\section{CONCLUSIONS}

Dermoid cysts are rare intracranial tumors. Traumatic rupture of the intracranial dermoid cyst is an extremely rare event. Symptoms of rupture can be variable and presentation can be delayed. CT scan is a good and fast option for diagnosis of the condition and ruling out other causes of similar clinical features at the same time especially a cerebrovascular accident. MRI is superior to CT scan for both diagnosis and treatment planning. Dissemination of fat droplets in the brain is considered diagnostic of a ruptured dermoid cyst. For treatment, each case has to be decided on its own merits, judging the risk-benefit ratio of neurosurgery over medical management.

\section{ACKNOWLEDGMENTS}

We would like to thank Dr. Jyotirmoy Das, Senior Resident, Department of Anesthesiology, North Bengal Medical College Hospital, Sushruta Nagar, and Dr. Abhijit Mukherjee, Associate
Professor, Department of Community Medicine, NRS Medical College, Kolkata for helping us throughout the process.

\section{REFERENCES}

1. Osborn AG, Hedlund GL, Salzman KL. Osborns Brain: Imaging, Pathology, and Anatomy. Philadelphia, PA: Elsevier; 2018.

2. Arseni C, Danaila L, Constantinescu AI, et al. Cerebral dermoid tumours. Neurochirurgia (Stuttg) 1976;19:104-14

3. Kim IY, Jung S, Jung TY, et al. Traumatic rupture of an intracranial dermoid cyst. J Clin Neurosci 2008;15:469-71.

4. Park SK, Cho KG. Recurrent intracranial dermoid cyst after subtotal removal of traumatic rupture. Clin Neurol Neurosurg 2012;114:421-4.

5. Phillips WE $2^{\text {nd }}$, Martinez CR, Cahill DW. Ruptured intracranial dermoid tumor secondary to closed head trauma. Computed tomography and magnetic resonance imaging. J Neuroimaging 1994;4:169-70.

6. Smith AS, Benson JE, Blaser SI, et al. Diagnosis of ruptured intracranial dermoid cyst: Value MR over CT. AJNR Am J Neuroradiol 1991;12:175-80.

7. Muçaj S, Ugurel MS, Dedushi K, et al. Role of MRI in diagnosis of ruptured intracranial dermoid cyst. Acta Inform Med 2017;25:141-4.

8. Stendel R, Pietilä TA, Lehmann K, et al. Ruptured intracranial dermoid cysts. Surg Neurol 2002;57:391-8.

9. Esquenazi Y, Kerr K, Bhattacharjee MB, et al. Traumatic rupture of an intracranial dermoid cyst: Case report and literature review. Surg Neurol Int 2013;4:80

10. Yasargil MG, Abernathey CD, Sarioglu AC. Microneurosurgical treatment of intracranial dermoid and epidermoid tumors. Neurosurgery 1989;24:561-7.

11. Kucera JN, Roy P, Murtagh R. Ruptured intracranial dermoid cyst manifesting as new onset seizure: A case report. J Radiol Case Rep 2011;5:10-8.

12. Lynch JC, Aversa A, Pereira C, et al. Surgical strategy for intracranial dermoid and epidermoid tumors: An experience with 33 patients. Surg Neurol Int 2014;5:163.

13. Kosuge Y, Onodera H, Sase T, et al. Ruptured dermoid cyst of the lateral cavernous sinus wall with temporary symptoms: A case report. J Med Case Rep 2016;10:224.

14. Mouskou S, Stamati A, Sfakianos G, et al. Ruptured intracranial dermoid cyst in a child. Cause of atypical neurological symptoms. Interdiscip Neurosurg 2020;20:100482.

15. Jamjoom AB, Cummins BH. The diagnosis of ruptured intracranial dermoid cysts. Br J Neurosurg 1989;3:609-12.

16. Liu JK, Gottfried ON, Salzman KL, et al. Ruptured intracranial dermoid cysts: Clinical, radiographic, and surgical features. Neurosurgery 2008;62:377-84.

Funding: None; Conflict of Interest: None Stated.

How to cite this article: Das U, Pandit N. Traumatic rupture of an intracranial dermoid cyst: A case report. Eastern J Med Sci. 2021;6(4):69-72.

DOI: $10.32677 /$ ejms.v6i4.3181 